\title{
Candidiasis oral en el paciente mayor
}

\section{Oral candidosis in the older patient}

\author{
Otero Rey E*, Peñamaría Mallón $M^{*}$, Rodríguez Piñón $M^{* *}$, Martín Biedma B***, \\ Blanco Carrión $A^{* * * * *}$
}

\section{RESUMEN}

La candidiasis o candidosis oral es la enfermedad infecciosa ocasionada por el crecimiento de las colonias de Cándida y la penetración de las mismas en los tejidos orales cuando las barreras físicas y las defensas del huésped se encuentran alteradas. Es una infección frecuente de la cavidad oral de los adultos de edad avanzada. Aunque la incidencia real se desconoce, se sabe que existe una prevalencia aumentada en ciertas ocasiones como ocurre en ancianos, en presencia de prótesis mucosoportadas, xerostomía o en patologías asociadas frecuentemente en los mayores. Los tipos clínicos más característicos son la forma seudomembranosa y la eritematosa (palatina y lingual). Pueden tener evolución aguda o crónica según la persistencia de los factores predisponentes. También son frecuentes procesos bucales comúnmente asociados: estomatitis protética, queilitis angular, glositis romboidal y lengua vellosa. La mayor parte de las candidiasis orales tienen un diagnóstico clínico, pero ha de confirmarse demostrando la penetración de la cándida en la mucosa oral, siendo el frotis la técnica de elección. Antes de comenzar el tratamiento, debemos estar seguros que se trata de una candidiasis oral, el tipo clínico y los factores predisponentes relacionados con la infección. Empezaremos siempre eliminando estos factores predisponentes, en el adulto mayor, la polifarmacología, la xerostomía, enfermedades crónicas y el uso de prótesis mucosoportadas son situaciones frecuentes que habrá que controlar. Instauraremos medidas higiénicas bucales y posteriormente si es necesario, utilizaremos fármacos antifúngicos, comenzando siempre con formas tópicas.

Palabras clave: Candidiasis oral, mucosa oral, paciente mayor, gerodontología.

\section{SUMMARY}

Oral Candidiasis or Candidosis is the infectious disease caused by the growth of colonies of Candida and penetration in the oral tissues when physical barriers and host defenses are altered. It is the most common fungal infection of oral involvement. It is a common infection of the oral cavity in elderly adults. Although the true incidence is unknown, it is known that there is an increased prevalence in certain situations in the elderly: tissue-borne prosthesis, xerostomia or disorders frequently associated. The most characteristic clinical types are pseudomembranous and erythematous (palatal and lingual) form. They may have acute or chronic evolution as the persistence of predisposing factors. They are also frequent mouth commonly associated processes: denture stomatitis, angular cheilitis, rhomboid glossitis and hairy tongue. Most oral candidiasis have a clinical diagnosis, but must be confirmed by demonstrating penetration of candida on the oral mucosa, being the preferred technique smears. Before starting treatment, we must be sure that it is an oral candidiasis, clinical type and predisposing factors associated with infection. Always start eliminating these predisposing factors in the elderly, the polypharmacy, xerostomia, chronic diseases and the use of tissue-borne prostheses are common

* Tutor Clínico. Profesor del Máster de Odontología Práctica Diaria de la Universidad de Santiago de Compostela.

** Profesor asociado de PTD de la Universidad de Santiago de Compostela.

*** Profesor Titular de PTD. Director de Máster de PTD de la Universidad de Santiago de Compostela.

**** Profesor Titular de Medicina Oral. Director de Máster de Odontología Práctica Diaria de la Universidad de Santiago de Compostela. 
situations which must be controlled. We will initiate oral hygiene measures and then if necessary, use antifungal drugs, always starting with topical forms.

Key words: Oral candidiasis, oral mucosa, older patient, gerodontology.

Fecha de recepción: 2 de mayo 2015.

Aceptado para publicación: 6 de mayo 2015.

Otero Rey E, Peñamaría Mallón M, Rodríguez Piñón M, Martín Biedma B, Blanco Carrión A. Candidiasis oral en el paciente mayor. Av. Odontoestomatol 2015; 31 (3): 135-148.

\section{INTRODUCCIÓN}

La candidiasis o candidosis oral es la enfermedad infecciosa ocasionada por el crecimiento de las colonias de Cándida y la penetración de las mismas en los tejidos orales cuando las barreras físicas y las defensas del huésped se encuentran alteradas. Es la infección micótica de afectación oral más frecuente (1). Dado que, entre todas las especies de Cándida, la que con mayor frecuencia ocasiona candidiasis es la $C$. albicans, numerosos clínicos consideran candidiasis oral como sinónimo de infección por C. albicans (1).

La presencia de especies del género Cándida en la cavidad oral es un hallazgo muy habitual (7-65\%). Sin embargo, muy pocos portadores sufren infecciones por Cándida. Además, las concentraciones de Cándida en portadores sanos son muy inferiores a las concentraciones halladas en personas que padecen distintas formas de candidiasis, 300-800 unidades formadoras de colonias por mililitro (UFC/ml) de saliva frente a recuentos superiores a 20.000 UFC/ $\mathrm{ml}$, respectivamente. Estos datos tienen un valor limitado dado que personas sanas pueden tolerar concentraciones altas de Cándida sin padecer la enfermedad, mientras que recuentos más bajos pueden precipitarla en personas debilitadas $(2,3)$. Se considera un patógeno oportunista, suele denominarse la "enfermedad de enfermos" (4). La candidiasis oral constituye un proceso frecuente. Se considera que más de 4/1.000 pacientes de una consulta general presentan signos de infección. No obstante, dado que la mayor parte de los casos cursan sin sintomatología aparente, la prevalencia debe ser mayor. La incidencia real de este proceso se desconoce, pero se sabe que existe una prevalencia aumentada en ciertas ocasiones: edades extremas (recién nacidos y ancianos), en presencia de prótesis mucosoportadas, xerostomía o en patologías asociadas (4).

A pesar de que la C. albicans es la especie oral más prevalente y la que con mayor frecuencia se convierte en patógena, se han conseguido aislar en el ser humano cerca de 20 géneros y casi 90 especies de levaduras (4). La mayoría son del género Cándida, aunque hay otras especies de hongos como la Rhodotorula glutinis y el Saccharomyces cerevisae, se encuentran en la boca ocasionalmente y no se conoce que causen infecciones. Lo mismo sucede con el Cryptococcus neoformans, que generalmente sólo se aísla en pacientes con criptococosis (4).

La mayoría de autores coinciden en que la colonización de la cavidad oral por hongos y más concretamente por C. albicans, es muy habitual entre personas sanas, y más aún en las personas mayores (entre un $7 \%$ y un 65\%). Los factores que afectan el estado de portador son la edad, el sexo, alteraciones salivales cuantitativas y cualitativas, el uso de prótesis mucosoportadas, el tabaco, el estado de salud, fundamentalmente alteraciones inmunológicas o endocrinas, determinados tratamientos farmacológicos, etc. Incluso se ha podido comprobar que existen variaciones del estado de portador a lo largo del día y una especial afinidad por colonizar el dorso lingual, el paladar y la mucosa bucal (4-6). En cuanto a la edad, los valores medios de prevalencia varían. En los neonatos, las cifras son relativamente bajas (16\%); se incrementan durante los 18 primeros meses de vida (44\%), disminuyen durante la infancia (6\%) para, de nuevo, subir en la edad adulta y, sobre todo, en la 
senectud. Por esto y por otros muchos factores: la hiposalivación, la existencia de prótesis removibles, alteraciones del sistema inmunitario y/o endocrino y la polimedicación, los ancianos son frecuentemente portadores de C. albicans (2).

\section{ETIOPATOGENIA}

Para que los C. albicans pase de su estado comensal a un estado patógeno, han de coincidir: factores de virulencia del hongo, alteración de los mecanismos de defensa frente a la infección candidiásica, existir una interacción huésped-microorganismo y la participación de unos factores predisponentes indispensables para que se produzca la infección. En trabajos se ha comprobado la imposibilidad de provocar infecciones por Cándida en mucosa oral intacta. Así, cuando a sujetos sanos se les inoculan organismos de Cándida, no desarrollan candidiasis. Es decir, deben existir una serie de factores que provoquen que el microorganismo se vuelva infectivo. Podemos hablar entonces de unos factores predisponentes o favorecedores de la candidiasis. Vamos a dividirlos en locales, sistémicos e iatrogénicos (3) (Tabla 1).

\section{CLÍNICA}

Una nota común en todas las clasificaciones ha sido la tendencia a diferenciar claramente las formas agudas, de corta evolución y que remiten con el tratamiento, de las formas crónicas, de larga evolución y generalmente rebeldes al tratamiento, probablemente por la persistencia de factores predisponentes. Las formas clínicas de candidiasis oral en el anciano son: candidiasis pseudomembranosa, candidiasis eritematosa, tanto de evolución aguda como crónica, candidiasis hiperplásica crónica, alteraciones orales comúnmente asociadas a candidiasis (palatitis subplaca, queilitis comisural, glositis romboidal y lengua vellosa) formas de candidiasis mucocutáneas crónicas en el anciano.

\section{Candidiasis pseudomembranosa}

Esta forma clínica, típica en lactantes (muguet), puede aparecer en ancianos debilitados o en personas mayores después de tratamientos con antibióticos y/o corticoides, con enfermedades malignas o bien en situaciones de alteración de los mecanismos inmunitarios. Pueden presentarse de forma aguda, menos de 15 días de evolución, o de forma crónica, persistiendo en el tiempo debido a que persisten los factores predisponentes. Se manifiesta en forma de placas blanquecinas o amarillentas, blandas y cremosas, semiadherentes, localizadas en cualquier parte de la mucosa bucal; lesiones en las que la $C$. albicans tiene un papel etiológico primario (2). Clásicamente, han sido descritas como copos de nieve o con el aspecto de coágulos de leche, fácilmente eliminados por frotamiento dejando áreas de mucosa normal o ligeramente eritematosa (Fig. 1). Este signo clínico permite realizar el diagnóstico diferencial con otras lesiones blancas de aspecto similar, como la leucoplasia o el liquen plano. La presencia de micelios abundantes en las muestras citológicas facilita el diagnóstico. A diferencia de los lactantes, en los que las placas blanquecinas cubren extensas áreas de mucosa, las lesiones blancas en los pacientes ancianos, se interponen entre lesiones eritematosas. La sintomatología suele ser muy escasa, aunque en ocasiones pueden relatar pérdida del gusto, mal sabor de boca y ardor, o incluso dolor (1).

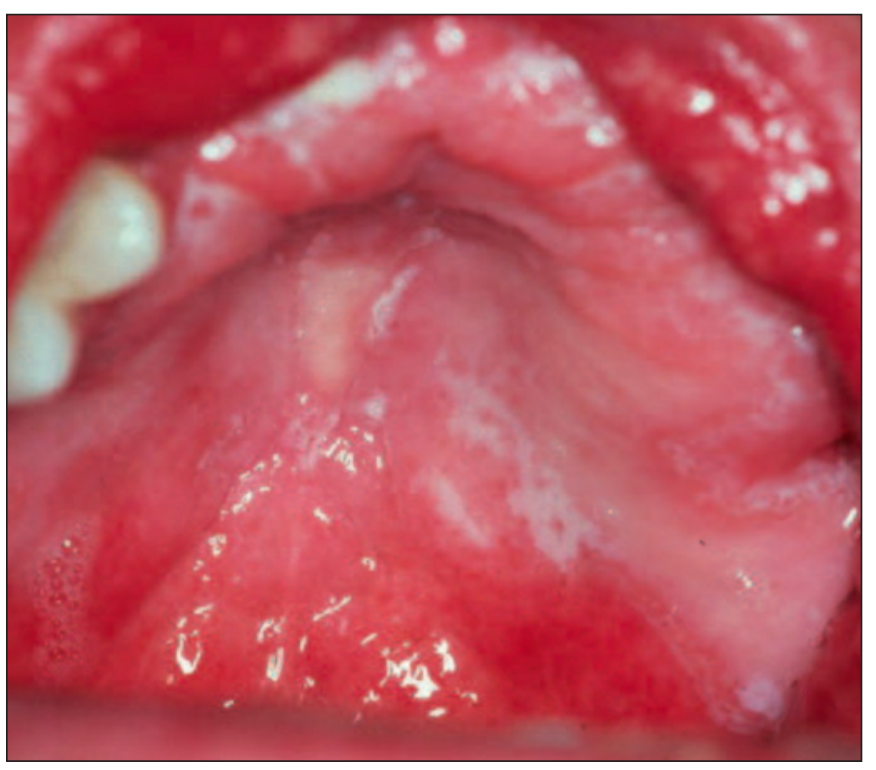

Fig. 1. Candidiasis seudomembranosa. Las placas blancas se desprenden al raspado. 


\section{TABLA 1.- FACTORES PREDISPONENTES EN LA INFECCIÓN POR CÁNDIDAS}

\section{Factores predisponentes locales}

\section{- Actúan sobre la barrera mucosa:}

- Pérdida de continuidad o integridad de la mucosa. En especial las prótesis mucosoportadas, muy frecuentes en los pacientes de edad avanzada (7), la simple fisuración del epitelio incluso en prótesis bien adaptadas puede ser suficiente (8). Las prótesis son un importante reservorio, y eso hace que en entre el 11 y el $77 \%$ de las estomatitis protéticas resultan en cultivo positivo. Otro presentación crónica relacionada con la edad y traumatismo repetidos es la queilitis angular (9).

- Modificaciones en el espesor de la mucosa. A medida que el epitelio se atrofia, un elemento más del envejecimiento se facilita la penetración de la cándida y la propia C. albicans es capaz de inducir cambios, en especial incrementado la actividad mitótica $(10,11)$.

- Alteraciones salivales. La disminución en calidad y cantidad está directamente relacionada con la sobreinfección (3,4) y de manera muy especial su acidificación (12).

- Flora comensal. El papel es dual, así lo demuestran diferentes estudios in vitro $(13,14)$.

- Dieta rica en hidratos de carbono. Aparte del efecto sistémico también actúa a nivel local, su metabolismo ocasiona una disminución del $\mathrm{pH}$ local y las concentraciones altas de glucosa aumentan los receptores $i \mathrm{C} 3 \mathrm{~b}$ en la C. albicans, incrementando su resistencia a la fagocitosis.

- Tabaco. Su influencia es controvertida, el humo del tabaco tiene algún factor anticándida en saliva; si bien, el hábito de fumar puede favorecer la aparición de lesiones en la mucosa que facilitan la colonización (5). El humo contiene nutrientes para la C. albicans, incluso algunas especies de Cándida pueden convertir los hidrocarburos aromáticos del humo en metabolitos carcinogénicos (15).

\section{Factores predisponentes sistémicos}

- Edad. La candidiasis oral, especialmente la palatitis candidiásica crónica y la queilitis angular, es una enfermedad frecuente en los ancianos, con una prevalencia de $38 \%$ y $26 \%$ respectivamente. Sin embargo, la tercera edad no debe considerarse un factor predisponente en sí, pero son pacientes mas polimedicados y con más patología sistémica (16)

- Alteraciones endocrinas. La diabetes es la más relacionada (17), dato no aceptado por todos los estudiosos. El mecanismo no está claro, la concentración de glucosa, la mejora en la facilidad de adhesión de hongo o el uso asociado de prótesis y tabaco. También el híper y el hipotiroidismo, así como el embarazo favorecen el desarrollo de la enfermedad. En este último caso, son los cambios hormonales los principales responsables (2).

- Alteraciones nutricionales. La anemia ferropénica es la mas frecuentemente implicada (18), pero no todos comparten esta idea (19), la etiopatogenia puede estar en la afectación que esta deficiencia ocasiona en el epitelio.

- Alteraciones del sistema inmune. En los pacientes con sida, ancianos o no, es la infección oportunista más frecuente, entre el 11 y el 96\% (20). Su aparición está directamente relacionada con niveles de CD4. También constituye un hallazgo habitual en determinados síndromes graves de inmunodeficiencia (síndrome de Di George, síndrome de Glanzmann-Riniker). Los cuadros de candidiasis mucocutánea también se relacionan con trastornos inmunológicos y/o endocrinos (1).

- Enfermedades sistémicas. Como comentamos anteriormente, la anemia ferropénica se asocia en numerosas ocasiones a candidiasis oral en sus distintas presentaciones clínicas (candidiasis mucocutánea crónica, candidiasis atrófica crónica, glositis atrófica y queilitis angular) (21). En ciertas enfermedades malignas, también es mayor la prevalencia. Las formas agudas de candidiasis orales son frecuentes en los pacientes con síndromes mieloproliferativos.

- Grupos sanguíneos. Es conocida la relación entre los grupos sanguíneos y la vulnerabilidad a las enfermedades infecciosas, incluidas las infecciones micóticas (22). El antígeno H funciona como un receptor para la C. albicans; por ello las personas con grupo O son más susceptibles a la colonización y posterior infección (22)

\section{Factores iatrogénicos}

- Tratamiento con antibióticos. El ejemplo más claro es el de la glositis candidiásica atrófica aguda o lengua dolorosa antibiótica. Puede suceder que, en condiciones normales, exista una competencia entre la Cándida y las bacterias por los nutrientes esenciales y/o por los receptores en la superficie de las células epiteliales (6). Por otro lado algunos antibióticos pueden actuar vía sistémica sobre la flora fúngica oral. Antibióticos como la eritromicina, el cotrimoxazol y algunos aminoglucósidos reducen la actividad anticándida de los neutrófilos in vitro, mientras que otros como la penicilina y la tetraciclina aumentan la respuesta inmune frente a la Cándida (23). Así mismo, el uso excesivo de enjuagues con antibacterianos también puede favorecer la infección oral por hongos (9).

- Tratamiento con corticoides. Diversos estudios han descrito que los corticosteroides de forma sistémica favorecen el crecimiento de la C. albicans. Sin embargo, al efecto de los corticoides debería añadirse el papel de la enfermedad de base para la cual se administran (24). La creciente utilización de corticoides vía inhalatoria, muy utilizada en los pacientes mayores, para el tratamiento de las formas leves o como coadyuvante en las formas más graves de asma se acompaña de un aumento de la prevalencia oral de Cándida y de una mayor predisposición a la infección por esta, pero la incidencia de candidiasis oral debida a esta causa es baja y carece de importancia clínica (25).

- Anticonceptivos y terapia sustitutiva. Existe la creencia general de que los anticonceptivos hormonales predisponen a candidiasis vaginal, pero no disponemos de estudios sobre la prevalencia oral (26).

- Tratamiento con quimio y/o radioterapia. En algunos centros, las infecciones fúngicas, en particular las candidiasis, constituyen la principal causa de muerte en los pacientes leucémicos sometidos a tratamiento con quimioterapia (27). La neutropenia asociada a la medicación y la toxicidad directa de los productos sobre la mucosa parecen las causas más directamente relacionadas con la sobreinfección. Así la frecuencia de candidiasis oral en los pacientes sometidos a tratamiento quimioterápico se ha cifrado en un 16\% para los casos de leucemia y en un 7\% para los pacientes con tumores sólidos (28). 


\section{Candidiasis eritematosa aguda}

Las lesiones aparecen como áreas de eritema, de mayor o menor tamaño, en la mucosa oral. Cualquier parte de la mucosa oral puede verse afectada, aunque, presenta cierta afinidad por localizarse en el dorso lingual (Fig. 2). Algunos autores consideran que puede aparecer de forma primaria o ser secundaria a la forma pseudomembranosa aguda. Otros, sin embargo, sólo reconocen la existencia de una forma primaria de la misma que afectaría al dorso lingual después de la administración indiscriminada de antibióticos de amplio espectro y/o corticoides (1). Cuando afecta al dorso de la lengua se produce una depapilación de la mucosa lingual acompañada de impotencia funcional, existe una imposibilidad para ingerir alimentos ácidos, picantes o calientes. Es la única variedad de candidiasis bucal que produce auténtico dolor. Por la sintomatología, la localización preferentemente lingual y la relación con el uso de antibióticos de amplio espectro o de larga duración (sobre todo amoxicilina con ácido clavulánico) se denomina también "lengua dolorosa antibiótica" (1).

\section{Candidiasis eritematosa crónica}

Incluye dos formas clínicas: la palatitis candidiásica eritematosa crónica y la glositis candidiásica eritematosa crónica.

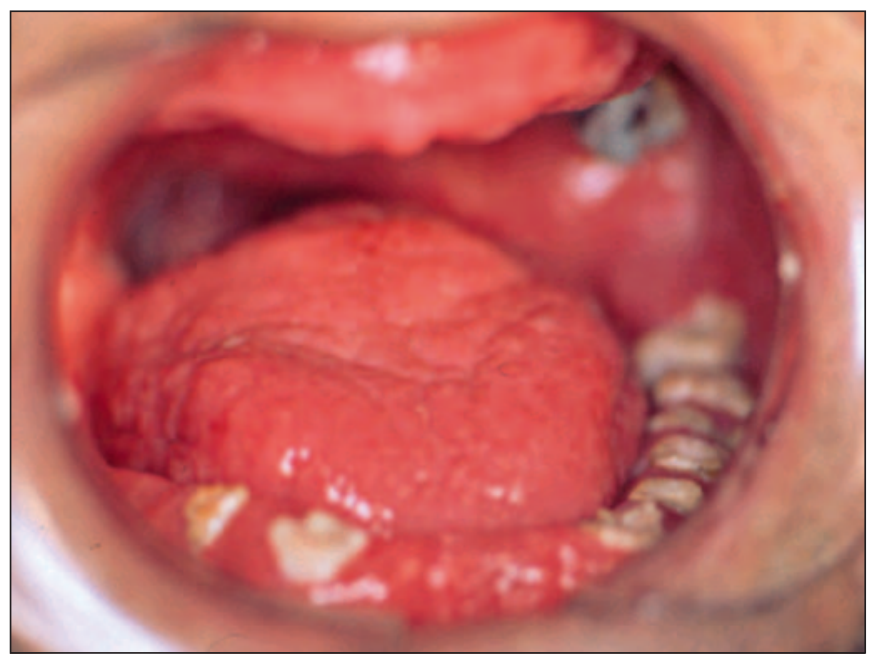

Fig. 2. Candidiasis eritematosa aguda o lengua dolorosa antibiótica.

\section{Palatitis candidiásica eritematosa crónica}

Relacionada con tres situaciones fundamentales: la inmunosupresión, infección por $\mathrm{VIH}$, y sobre todo, y en el caso de ancianos, enfermedades pulmonares crónicas que cursan con boca seca y la utilización de aerosoles y/o sprays (EPOC, asma, etc) y la sobreinfección candidiásica de una estomatitis por prótesis, en cuyo caso hablamos de estomatitis protética o estomatitis por prótesis asociada a Cándida $(1,16)$. Clínicamente aparece la mucosa palatina enrojecida, con atrofia de la mucosa afectada, ya sea parcialmente o todo el paladar. Es asintomático, pudiendo presentar alteraciones del gusto o mal sabor de boca. No suele ser motivo de consulta (Fig. 3).

\section{Glositis candidiásica eritematosa crónica}

Se manifiesta como áreas más o menos extensas en la superficie dorsal de la lengua, en la que han desaparecido las papilas filiformes, dando lugar a una superficie lisa de coloración rojiza (Fig. 4). Se relaciona con dos situaciones fundamentales, a veces coincidentes, con xerostomía y asociada a la palatitis candidiásica eritematosa crónica. En este último caso se dice que da una imagen clásica en calcado o en espejo. La sintomatología es también escasa, hay una alteración del gusto que no se acompaña de dolor, a lo sumo un ligero escozor (1).

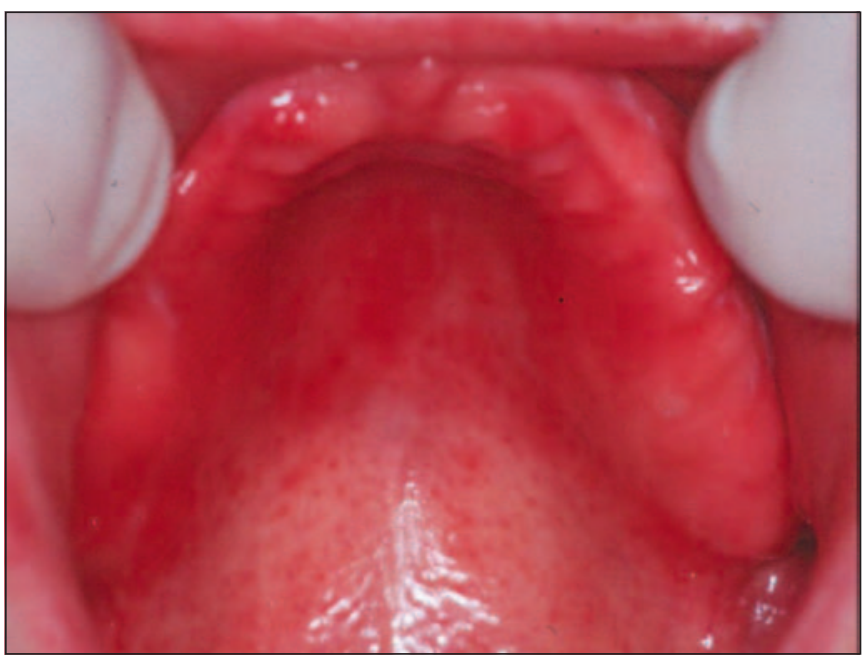

Fig. 3. Candidiasis eritematosa crónica del paladar. 


\section{Candidiasis hiperplásica crónica o leucoplasia-candidiasis}

Es una forma de candidiasis poco frecuente. Se caracteriza por la presencia de placas blancas que no se desprenden con el raspado, persistentes en el tiempo y que se localizan por orden de frecuencia en mucosas yugales (sobre todo en zona retrocomisural), lengua, labios y paladar. A menudo las lesiones son bilaterales, retrocomisurales con forma triangular de base anterior y vértice posterior. Se distinguen dos formas: la forma homogénea, que se presenta como una placa blanca, uniforme, adherente y asintomática, y la forma nodular, dolorosa, caracterizada por la presencia de nódulos múltiples blanquecinos en una mucosa eritematosa (1). Es la única forma de candidiasis en la que está indicada la biopsia para diferenciarla de otros procesos. Clínica e histológicamente es indistinguible de una leucoplasia. Sólo una buena respuesta al tratamiento antifúngico confirma el diagnóstico.

\section{Lesiones orales comúnmente asociadas a candidiasis}

\section{Estomatitis protética}

La estomatitis protética, también denominada estomatitis subprótesis o palatitis subplaca, es una entidad clínica que aparece en portadores de prótesis

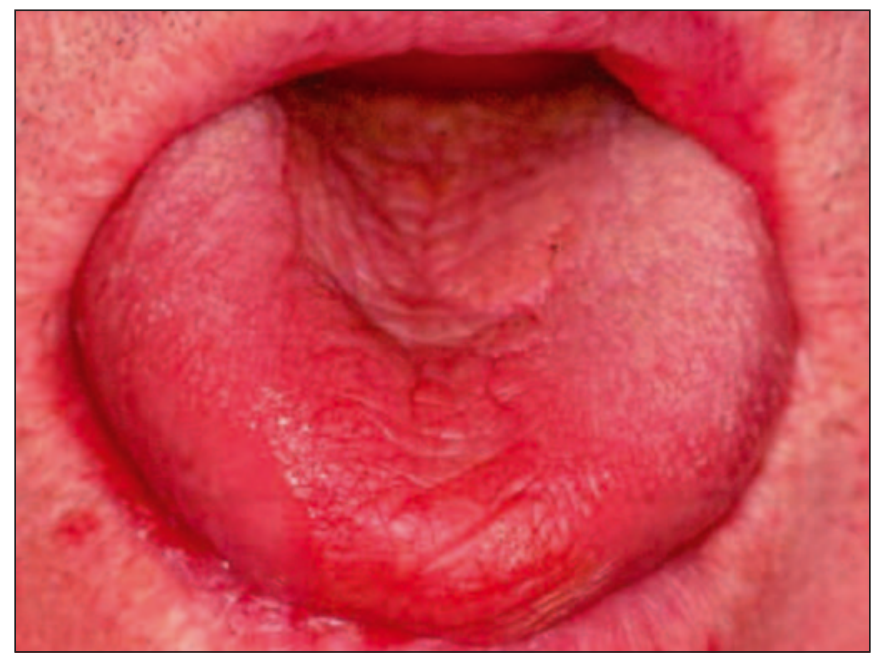

Fig. 4. Glositis candidiásica eritematosa crónica. mucosoportadas, completas o parciales, que se caracteriza por alteraciones eritematosas en la mucosa sobre la que asientan (Fig. 5). Aunque puede afectar a la mucosa mandibular, la más frecuentemente afectada es la mucosa palatina. La media de edad de los pacientes está en torno a los 50 años. En cuanto al sexo, se ha observado una mayor prevalencia entre la población femenina (16). Es una lesión traumática y en su origen se implican además diversos factores como una higiene deficiente, la utilización sin descanso de la prótesis, microtraumatismos continuados sobre la mucosa, por un desajuste o mala adaptación de la prótesis, etc. Su prevalencia entre los portadores de prótesis removible es muy variable (11$77,4 \%$ ); y dentro de estos, es más frecuente en los portadores de prótesis completas que en los portadores de prótesis parciales. Por otro lado, la presencia de cándida en pacientes con estomatitis por prótesis y en portadores de prótesis sin evidencia clínica de estomatitis es similar (93/86\%). Sin embargo, en los pacientes con estomatitis protética hay una mayor densidad de levaduras (UFC/ml) en la superficie de la prótesis en contacto con la mucosa palatina y en dicha mucosa, los recuentos de colonias fúngicas son 100 veces superiores en los pacientes con estomatitis $(0,3 \%)$ frente a los obtenidos en pacientes con una mucosa palatina intacta $(0,002 \%)(56)$. De todos modos, en la etiopatogenia de la estomatitis protética, se encuentran implicadas tanto colonias fúngicas como bacterianas. Las colonias fúngicas habitualmente representan un porcentaje inferior

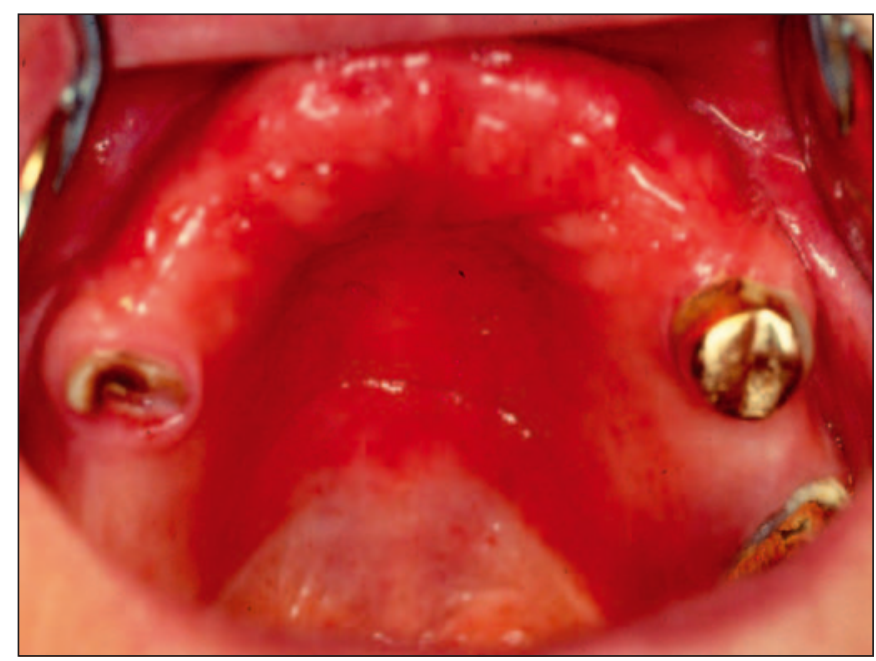

Fig. 5. Estomatitis protética con sobreinfección candidiásica. 
al $1 \%$ de la flora microbiana presente en la prótesis, aún en los casos de estomatitis protética y en ocasiones, sólo se aíslan bacterias. Por otro lado, el significado etiológico de un hallazgo positivo debe valorarse en función del lugar de toma de muestra, el tipo de Cándida, la presencia de otros microorganismos patógenos, las características clínicas y la existencia de factores predisponentes. De ahí que el diagnóstico de estomatitis por prótesis asociada a Cándida debe confirmarse por la demostración de levaduras en grandes cantidades, fundamentalmente a través de un frotis, tomadas de la mucosa subyacente e incluso de la prótesis. No es frecuente la invasión del tejido por hifas; es más probable que las especies de Cándida provoquen la disolución enzimática de la superficie epitelial para favorecer la penetración de antígenos y toxinas y así desencadenar una respuesta inflamatoria. Sin embargo, a pesar de la pequeña proporción de levaduras aisladas en la placa dental, la estomatitis por prótesis mejora tras el tratamiento con antimicóticos. Es por ello que las levaduras deben considerarse como patógenos oportunistas de gran importancia en el desarrollo de la estomatitis (16).

Dentro de las especies de Cándida, la C. albicans es la que con más frecuencia se asocia con la estomatitis por prótesis; particularmente el serotipo A, mientras que en el resto de la cavidad oral se aíslan ambos serotipos (A y B) (2). La higiene deficiente o la diabetes son factores predisponentes (17) así como prótesis deterioradas o mal ajustadas. El proceso se inicia con un eritema y edema que afecta a parte o a la totalidad de la mucosa palatina que es asintomático $\mathrm{y}$, a lo sumo, el paciente refiere episodios dolorosos caracterizados por ligeras molestias. En estadios más avanzados, se forman zonas de hiperplasia y formaciones nodulares que podrían ulcerarse por acción del trauma protético, dando entonces mayor sintomatología. Según la extensión y severidad del proceso pueden distinguirse tres categorías (Newton, 1962):

a) Tipo I de Newton: Localizado y caracterizado por un punteado rojizo sobre la mucosa palatina.

b) Tipo II de Newton: Lesión eritematosa generalizada que afecta a parte o a toda la mucosa cubierta por la prótesis.

c) Tipo III de Newton: Es un tipo granular (hiperplasia papilar inflamatoria) que afecta a la parte central del paladar duro y bordes alveolares.
El tipo I se relaciona con la obstrucción de los ductus salivales por la prótesis, mientras que los tipos II y III se relacionan con el acúmulo de placa microbiana (bacteriana o fúngica) en la prótesis y en la mucosa subyacente.

Es importante considerar que la estomatitis por prótesis asociada a Cándida no es una condición grave en personas sanas. La mayor parte de los casos responden bien al tratamiento con antifúngicos pero las recidivas son frecuentes y la infección tiende a extenderse a otras partes de la mucosa oral. En personas debilitadas pueden ser el origen de infecciones sistémicas, especialmente en sujetos sometidos a radioterapia del área orofaríngea o a tratamientos prolongados con antibióticos, corticoides o inmunosupresores. En cuanto al desarrollo de reacciones a los diferentes constituyentes de las bases protésicas, es un hecho bastante infrecuente $(16,30)$.

\section{Queilitis comisural o angular por Cándidas}

También llamada "boquera" o "perleche". Es el diagnóstico clínico de lesiones que afectan a los ángulos de la boca. Son lesiones generalmente bilaterales en las comisuras, caracterizadas por pequeñas erosiones, fisuras y grietas con formaciones costrosas a su alrededor (Fig. 6). La sintomatología varía de dolor intenso con gran afectación de la capacidad funcional, a escasa (1). En las lesiones se aíslan frecuentemente levaduras y estafilococos, y desaparecen tras el tratamiento con antimicrobianos, pero el hecho de que las lesiones recidiven tras el cese del trata-

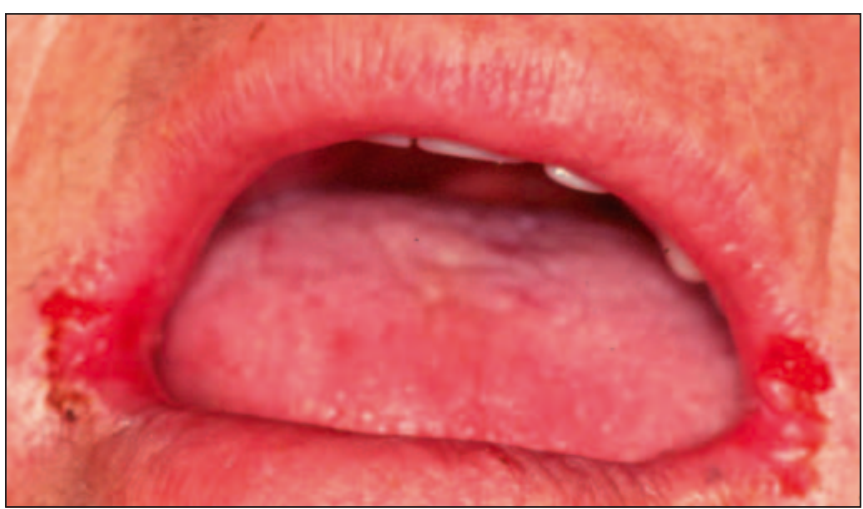

Fig. 6. Queilitis angular bilateral. 
miento médico hace pensar que existe una serie de factores predisponentes locales o sistémicos a los que la infección es secundaria. Entre estos factores destacan la pérdida de la dimensión vertical (circunstancia que frecuentemente se produce en portadores de prótesis) y déficits vitamínicos, sobre todo de riboflavina, hierro y ácido fólico. En ocasiones la queilitis angular se observa junto a la estomatitis por prótesis asociada a Cándida, a menudo indica la progresión de la infección por Cándida desde la mucosa cubierta por la prótesis hacia los ángulos de la boca (16). Ohman y cols. (1985) (31) la clasificaron en cuatro grupos:

a) Tipo I: Localizada, con lesión mínima en piel.

b) Tipo II: Fisurada, con rágades, más extensa en longitud y profundidad.

c) Tipo III: Con fisuras intensas en forma radial desde el ángulo a la piel.

d) Tipo IV: Eritematosa, sin fisuras. Se extiende al borde de los labios.

El tipo I se observa con mayor frecuencia en los pacientes dentados, mientras que los restantes tipos aparecen característicamente en pacientes portadores de prótesis.

\section{Glositis romboidal media}

Para algunos autores, la infección por Cándidas es la responsable. Fue descrita por Brocq (1907) con el nombre de glositis losángica mediana. Es una alteración relativamente rara de la lengua $(0,2-3,0 \%)$; más frecuente en varones. Existen cambios histopatológicos característicos, pero el diagnóstico suele ser clínico: se presenta en la línea media del dorso, por delante de las papilas circunvaladas, en forma de área rojiza, romboidal, plana y algunas veces mamelonada, que puede sobresalir de 2 a $5 \mathrm{~mm}$ de la superficie y en la que no se observan papilas filiformes, en algunas series es más prevalente en personas mayores (32). Clásicamente se ha considerado una anomalía congénita debido a la persistencia del tubérculo impar pero la baja frecuencia de lesiones a edades infantiles y la ausencia de una historia familiar de glositis romboidal media restó importancia a esta teoría. Después se postuló que podría ser la consecuencia de una infección crónica por C. albicans, favorecida por el hábito de fumar, pequeños traumatismos o por prótesis y muchos autores apoyaron la teoría de que, en realidad, se trataba de una forma crónica de candidiasis oral (33). Histológicamente se caracteriza por ausencia de las papilas filiformes, infiltrado inflamatorio, predominantemente de estirpe linfocitaria, e hiperplasia de la capa espinosa. Pueden observarse hifas en la zona paraqueratinizada lingual y en la capa superficial espinosa del epitelio (1).

\section{Lengua negra vellosa}

Es otra entidad relacionada con la infección por $C$. albicans. Es bastante frecuente en el anciano, sobre todo de sexo masculino. Se debe a un aumento de tamaño de las papilas filiformes en cuyo extremo se forman unas vellosidades que adoptan una coloración oscura, debido a la oxidación de la queratina. En un principio suele afectar al tercio medio de la lengua, pero puede extenderse por toda su superficie. El uso de antibióticos y antisépticos (clorhexidina) altera la ecología del medio oral contribuyendo al desarrollo de esta alteración. Los microorganismos saprofitos proliferan considerablemente, entre ellos la Cándida, además de bacterias con capacidad pigmentaria. La lengua negra vellosa puede ser también una complicación de la quimio y/o radioterapia, donde la disminución de la capacidad defensiva del huésped permite el desarrollo de los hongos $(34,35)$.

\section{Candidiasis mucocutánea crónica}

De las candidiasis mucocutáneas crónicas, la única que puede aparecer en personas mayores es la forma difusa. Se inicia tardíamente (a partir de los 55 años) y es la forma menos frecuente de todas. No tiene carácter hereditario y la candidiasis es la única manifestación de la enfermedad, que se manifiesta ocupando extensas áreas de piel, mucosa oral y uñas $(1,35)$.

\section{DIAGNÓSTICO}

La clínica es fundamental y el diagnóstico de laboratorio se basa en la demostración e identificación del 
hongo en las muestras clínicas (frotis o cultivo) y/o el diagnóstico serológico. Sin embargo, dado que la Cándida es un comensal habitual en la cavidad oral, su mera demostración microbiológica no es un dato significativo en ausencia de clínica sugestiva de candidiasis; es preciso, por ello, establecer el significado clínico del aislamiento del microorganismo. Tampoco se ha establecido un valor definitivo para el recuento de colonias que, de esta forma, permita diferenciar entre comensalismo y enfermedad. De ahí la necesidad de consensuar la cínica y el laboratorio para llegar a un diagnóstico de candidiasis oral (36).

Por tanto, basándonos en datos clínicos de sospecha de candidiasis, podemos confirmar el diagnóstico mediante microbiología (frotis y cultivo), histopatología y serología (Tablas 2 y 3 ).

\section{TRATAMIENTO}

Primero nos hemos de preguntar:

1. ¿Estamos seguros que es una candidiasis? La respuesta está en el diagnóstico, debe haber una confirmación clínica y microbiológica o histológica.

2. ¿Qué tipo clínico es? Según los datos clínicos que presente.

3. ¿Por qué? Buscaremos los factores predisponentes relacionados con la aparición de la candidiasis.

El tratamiento de las candidiasis orales requiere en primer lugar, la eliminación o atenuación de estos factores; siendo éste el aspecto fundamental en la terapéutica de esta infección en el anciano; y sin el

\section{TABLA 2.- DIAGNÓSTICO DE LA CANDIDIASIS}

\section{Sospecha clínica. Es fundamental}

\section{Microbiología:}

- El frotis citológico (mediante raspado o hisopo) suele ser útil. Se extiende el material obtenido en un porta, se pueden tratar con una solución de $\mathrm{KOH}$ (hidróxido de potasio) al $20 \%$ y posterior observación microscópica. Otras veces, la observación se realiza después de teñir el material de frotis con PAS, GRAM, Hematoxilina-Eosina o Papanicolau (65). De esta manera se identifica la C. albicans por observación de las células fúngicas (blastoporos con o sin hifas o pseudohifas).

- La identificación se basa en el cultivo. Un medio muy utilizado es el de agar de peptona-glucosa (dextrosa) o peptona-maltosa que, al ser descrito en 1896 por Sabouraud, adoptó su nombre. Hay medios específicos que permiten diferenciar colorimétricamente a especies concretas de Cándida (Microstix-Cándida ${ }^{\circledR}$, Oricult_N$N^{\circledR}$ ). Recientemente, se ha introducido el medio de CROMagar; este contiene un sustrato cromogénico que permite diferenciar las colonias de C. albicans, C. krusei y C. tropicalis, las cuales crecen de color verde, rosa pálido y azul grisáceo, respectivamente (37).

Histología: Sólo se debe realizar una biopsia en aquellas lesiones en las que exista un diagnóstico diferencial difícil, en los casos de candidiasis hiperplásica, cuando existen dudas manifiestas en el diagnóstico y cuando el proceso no responda al tratamiento correcto (68). Pueden pasar desapercibidas en una tinción rutinaria de Hematoxilina-Eosina. Por ello, se recomienda la tinción con PAS, plata metenamina, GRAM o Gomori para identificar los elementos fúngicos.. No permite, sin embargo, diferenciar la especie. La demostración de la invasión de tejidos por elementos fúngicos constituye criterio diagnóstico; es importante considerarlo porque la Cándida es un comensal habitual de la cavidad oral, tanto en humanos como en investigación animal $(38,39)$.

Inmunología: Lehner (71) fue, probablemente, el primero en utilizar técnicas de inmunofluorescencia para detectar la presencia de C. albicans. En la actualidad, pueden detectarse anticuerpos séricos (IgM, IgA, IgE) mediante inmunofluorescencia indirecta y test ELISA. Sin embargo, la utilidad de este tipo de técnicas, pudiendo utilizarse otras más simples y fiables queda en entredicho. De hecho, prácticamente no se emplean para el diagnóstico de laboratorio de candidiasis oral (40). 


\section{TABLA 3.- PROPUESTA DIAGNÓSTICA}

- En la candidiasis pseudomembranosa, la técnica de elección será un frotis. Se puede realizar un cultivo, pero no será definitivo.

- En la candidiasis eritematosa, para confirmar el diagnóstico habrá que determinar microbiológicamente la presencia del hongo. Para ello se debe realizar un frotis. Con el cultivo podemos tener falsos positivos.

- En la candidiasis hiperplásica, los frotis pueden demostrar abundantes hongos y células inflamatorias. Se recomienda realizar una biopsia siempre, ya que hay que descartar la existencia de fenómenos displásicos en el epitelio.

- En los pacientes con estomatitis por prótesis, el diagnóstico es también clínico. Las pruebas complementarias (frotis) tienen que confirmar la posible participación de la Cándida en el proceso para poder definirlo como candidiasis.

- La queilitis angular tiene un diagnóstico clínico. La sobreinfección por Cándida habrá que demostrarla mediante un frotis.

- En la glositis rómbica, el diagnóstico va a ser eminentemente clínico. Sólo se deberá realizar una biopsia en aquellas situaciones en las que se sospeche la presencia de un proceso tumoral.

- En la lengua negra vellosa, se hará un diagnóstico clínico. La presencia de cándida se confirmará mediante un frotis.

cual, probablemente se produzca una recidiva de las lesiones tras el cese de la terapia antimicótica aplicada $(1,30,41)$. Por oro lado es fundamental la puesta en marcha de una serie de medidas de carácter higiénico previas al tratamiento farmacológico. Utilizaremos soluciones alcalinas antisépticas suaves (bicarbonato, borato de sodio, hidróxido de magnesio) para el lavado de la cavidad oral y que dificultan la colonización y el crecimiento de los hongos. Otras sustancias colorantes, como el violeta de genciana 0,5-1\%, actúan también sobre los gérmenes gram + (1). En el caso de la estomatitis por prótesis, además del reposo nocturno sin prótesis, se aconseja la des- infección de la misma (16,30); el antiséptico de elección es el digluconato de clorhexidina al 0,2-0,12\%.

Posteriormente se comienza el tratamiento mediante una terapia antifúngica, tanto por vía local o tópica como por vía general. La decisión de tratar las infecciones superficiales con un agente tópico o sistémico depende del hongo, de su localización y de la extensión de la lesión. En general, los casos más simples se tratan de forma tópica, mientras que las formas más severas de la enfermedad se tratan por vía sistémica. El tratamiento tópico requiere un tiempo de contacto suficiente entre el fármaco y la mucosa oral. A fin de evitar recidivas, se recomienda continuar con la terapia 2-3 semanas más allá del cese de los signos y síntomas $(30,41)$.

La terapia con antimicóticos tópicos tras la eliminación (en lo posible) de los factores predisponentes, es la manera más efectiva de tratar la candidiasis oral en ancianos. Los agentes tópicos están disponibles en forma de enjuagues orales, tabletas orales, tabletas vaginales y cremas. Las distintas formas de aplicación pueden combinarse para aumentar el efecto tópico. En general, los enjuagues orales proporcionan un menor tiempo de contacto del fármaco y por ello una menor eficacia. Se usan, preferentemente, en los pacientes con sequedad oral, que tienen dificultades para disolver las formas en tableta. Las tabletas podrían ser la forma más efectiva de medicación puesto que se disuelven lentamente en la boca y proporcionan un mayor tiempo de contacto con el medio bucal y la faringe, pero son complejas de utilizar en la boca seca y además suelen endulzarse para mejorar el sabor (41). Para mejorar el contacto podemos utilizar adhesivos como el orabase, chitosán, zilactín o goma aguar. Se ha podido comprobar experimentalmente que la asociación de nistatina a orabase o chitosán produce una mejoría significativa, tanto a nivel clínico, microbiológico como histológico, de las lesiones causadas por la C. albicans en comparación con la nistatina sola (42). Es más, de los dos anteriores, el chitosán ha demostrado potenciar con mayor intensidad el efecto terapéutico de la nistatina, lo que se manifiesta especialmente en una mayor supresión de la colonización por $C$ albicans en la mucosa del dorso lingual, pero es un terreno en el que se está investigando de manera muy activa (43). 
Los antifúngicos tópicos normalmente utilizados son: nistatina, anfotericina B, y derivados azólicos (miconazol, clotrimazol, econazol y ketoconazol).

Cuando los agentes tópicos no son suficientes para controlar la infección, hay que recurrir a los agentes sistémicos. El uso concomitante de un agente tópico facilita una curación más pronta de la infección y permite reducir la dosis y la duración de la terapia sistémica. Las drogas sistémicas por vía oral se usan frecuentemente en los tratamientos ambulatorios; las candidiasis sistémicas en pacientes inmunodeprimidos requieren generalmente medicación por vía intravenosa (en centros hospitalarios). Los antifúngicos sistémicos más usados son ketoconazol, fluconazol, itraconazol, miconazol, anfotericina B, flucitosina y griseofulvina $(41,44,45)$. Repasemos algunos aspectos:

- Ketoconazol. Se utiliza en comprimidos de 200 mg, 1 o 2 diarios durante 3-4 semanas, siendo aún más efectivo en candidiasis genital que oral. Cabe destacar su hepatotoxicidad con elevación de transaminasas y a veces dolor abdominal y prurito. Por otra parte, la absorción depende de una adecuada secreción gástrica, por lo cual debe evitarse la administración simultánea de fármacos que inhiban la secreción gástrica o su acidez (anticolinérgicos, antiácidos, antagonistas $\mathrm{H}_{2}$ ) así como de otros fármacos cuya eficacia pueda verse reducida (antituberculosos, teofilina, etc.) (41).

- Fluconazol e itraconazol. El fluconazol es posiblemente el antifúngico sistémico de elección. Inhiben las enzimas asociadas al citocromo P 450 y bloquean la síntesis de ergosterol. Se usan en comprimidos con dosis de 50-200 mg/día de 1 a 4 semanas. Poseen baja toxicidad, en alguna ocasión náuseas, vómitos, diarrea y dolor de cabeza. Son más potentes que el ketoconazol pero bastante más caros. En ocasiones, el fluconazol se ha utilizado preventivamente en casos de inmunosupresión para evitar recidivas, pero consideramos que este podría ser el motivo de la aparición de resistencias $(39,41,46)$.

- Miconazol. En situaciones de candidiasis sistémicas puede usarse miconazol por vía oral o intravenosa. Es poco utilizado $(41,47)$.

- Anfotericina B. Se utilizará en candidiasis graves y se administrará por vía intravenosa $(41,48)$.
- Flucitosina. Análogo del fluoracil que se incorpora en el ARN fúngico, dando lugar a una deficiente síntesis de proteínas; bloquea la síntesis de ADN fúngico. Su poder antifúngico se conoce desde hace años (49). Desarrolla resistencias cuando se utiliza como único antifúngico, de ahí que se use frecuentemente en combinación con la anfotericina B (50).

- Griseofulvina. Acción fungostática. Interfiere la mitosis y la síntesis de la pared celular y los ácidos nucleicos. Tiene bastantes efectos secundarios. Contraindicada durante el embarazo. Se liga a la queratina de los tejidos de desarrollo. Actualmente no es un antimicótico de uso habitual

\section{BIBLIOGRAFÍA}

1. Singh A, Verma R, Murari A, Agrawal A. Oral candidiasis: An overview. J Oral Maxillofac Pathol 2014; 18:S81-5.

2. Update on oral fungal infections. Muzyka BC, Epifanio RN. Dent Clin North Am 2013;57:561-81.

3. Challacombe SJ. Immunologic aspects of oral candidiasis. Oral Surg Oral Med Oral Pathol 1994; 78: 202-10.

4. Cannon RD, Holmes AR, Mason AB, Monk BC. Oral Candida: Clearance, Colonization, or Candidiasis? J Dent Res 1995;74:1152-61.

5. Semlali A, Killer K, Alanazi H, Chmielewski W, Rouabhia M. Cigarette smoke condensate increases $\mathrm{C}$. albicans adhesion, growth, biofilm formation, and EAP1, HWP1 and SAP2 gene expression. BMC Microbiol 2014;14:61

6. Lynge Pedersen AM1, Nauntofte B, Smidt D, Torpet LA. Oral mucosal lesions in older people: relation to salivary secretion, systemic diseases and medications. Oral Dis. 2015 [Epub ahead of print].

7. Ishikawa KH, Mayer MP, Miyazima TY, Matsubara VH, Silva EG, Paula CR, et al. A multispecies probiotic reduces oral Candida colonization in denture wearers. J Prosthodont 2015;24:194-9. 
8. Tay LY, Jorge JH, Herrera DR, Campanha NH, Gomes BP, Andre Dos Santos F. Evaluation of different treatment methods against denture stomatitis: a randomized clinical study. Oral Surg Oral Med Oral Pathol Oral Radiol 2014;118:72-7.

9. Sharon V, Fazel N. Oral candidiasis and angular cheilitis. Dermatol Ther 2010;23:230-42.

10. McManus BA, Coleman DC. Molecular epidemiology, phylogeny and evolution of Candida albicans. Infect Genet Evol 2014;21:16678.

11. Patil PB, Bathi R, Chaudhari S. Prevalence of oral mucosal lesions in dental patients with tobacco smoking, chewing, and mixed habits: A crosssectional study in South India. J Family Community Med 2013 ;20:130-5.

12. Napeñas JJ, Rouleau TS. Oral complications of Sjögren's syndrome. Oral Maxillofac Surg Clin North Am 2014;26:55-62.

13. Makrides HC, MacFarlane TW. Effect of commensal bacteria on the adherence of Candida albicans to epithelial cells in vitro. Microbios Lett 1982; 21: 55-61.

14. Ishijima SA, Hayama K, Burton JP, Reid G, Okada M, Matsushita Y, Abe S. Effect of Streptococcus salivarius K12 on the in vitro growth of Candida albicans and its protective effect in an oral candidiasis model. Appl Environ Microbiol 2012;78: 2190-9.

15. Sanjaya PR, Gokul S, Gururaj Patil B, Raju R. Candida in oral pre-cancer and oral cancer. Med Hypotheses 2011;77:1125-8.

16. Martori E, Ayuso-Montero R, Martínez-Gomis J, Viñas $M$, Peraire $M$. Risk factors for denturerelated oral mucosal lesions in a geriatric population. J Prosthet Dent 2014;111:273-9.

17. Fatahinia M, Poormohamadi F, Zarei Mahmoudabadi A. Comparative Study of Esterase and Hemolytic Activities in Clinically Important Candida Species, Isolated From Oral Cavity of
Diabetic and Non-diabetic Individuals. Jundishapur J Microbiol 2015;8:e20893.

18. Samad A, Mohan N, Balaji RV, Augustine D, Patil SG. Oral manifestations of plummer-vinson syndrome: a classic report with literature review. J Int Oral Health 2015;7:68-71.

19. Samad A, Mohan N, Balaji RV, Augustine D, Patil SG. Oral manifestations of plummer-vinson syndrome: a classic report with literature review. J Int Oral Health2015;7:68-71.

20. Petruzzi MN, Cherubini K, Salum FG, Figueiredo MA. Risk factors of HIV-related oral lesions in adults. Rev Saude Publica 2013;47:52-9.

21. Ueng SW, Lee CY, Hu CC, Hsieh PH, Chang Y. What is the success of treatment of hip and knee candidal periprosthetic joint infection? Clin Orthop Relat Res 2013;471:3002-9.

22. Khozeimeh F, Mohammadpour M, Taghian M, Naemy V. A comparative study of Candida albicans mean colony counts and blood group antigens in the saliva of healthy subjects. Dent Res J (Isfahan) 2014;11:240-3.

23. Dolmer JE, Hector RF. Enhanced immune responses in mice treated with penicillintetracycline or trimethoprim-sulfamethoxazole when colonized intragastrically with Candida albicans. Antimicrob Agents Chemoth 1987;31: 691-7.

24. Garg A, Singh S. Enhancement in antifungal activity of eugenol in immunosuppressed rats through lipid nanocarriers. Colloids Surf B Biointerfaces 2011;87:280-8.

25. Matera MG, Cardaci V, Cazzola M, Rogliani P. Safety of inhaled corticosteroids for treating chronic obstructive pulmonary disease. Expert Opin Drug Saf 2015;14:533.

26. Dutt P, Chaudhary S, Kumar P. Oral health and menopause: a comprehensive review on current knowledge and associated dental management. Ann Med Health Sci Res 2013;3:320-3. 
27. Kishel JJ, Sivik J. Breakthrough invasive fungal infection in an immunocompromised host while on posaconazole prophylaxis: an omission in patient counseling and follow-up. J Oncol Pharm Pract 2008; 14:189-93.

28. Schelenz S, Abdallah S, Gray G, Stubbings H, Gow I, Baker P, et al. Epidemiology of oral yeast colonization and infection in patients with hematological malignancies, head neck and solid tumors. J Oral Pathol Med 2011;40:83-9.

29. Theilade E, Budtz-Jörgensen E. Predominant cultivable microflora of plaque on removable dentures in patients with denture-induced stomatitis. Oral Microbiol Immunol 1988;3:8-13.

30. Ayuso-Montero R, Torrent-Collado J. López-López J. Estomatitis protésica: puesta al día. RCOE 2004;9:657-62.

31. Ohman SC, Dahlén G, Möller A, Ohman A. Angular cheilitis: a clinical and microbial study. J Oral Pathol 1986;15:213-7.

32. Pili FM, Erriu M, Piras A, Garau V. Application of the novel method in the diagnosis and treatment of median rhomboid glossitis Candida-associated. Eur J Dent 2014;8:129-31.

33. Goregen M, Miloglu O, Buyukkurt MC, Caglayan F, Aktas AE. Median rhomboid glossitis: a clinical and microbiological study. Eur J Dent 2011;5: 367-72.

34. López J, Jané E, Chimenos E, Roselló X. Actualización de la candidiasis oral. Arch Odontoestomatol 1997;13:259-72.

35. Thompson DF, Kessler TL. Drug-induced black hairy tongue. Pharmacotherapy 2010;30:585-93.

36. Stoopler ET, Sollecito TP. Oral mucosal diseases: evaluation and management. Med Clin North Am 2014;98:1323-52.

37. Albaina O, Sahand IH, Brusca MI, Sullivan DJ, Fernández de Larrinoa I, Moragues MD. Identification and characterization of nine atypical Candida dubliniensis clinical isolates. J Med Microbiol 2015;64:147-56.
38. Montal S, Bousquet P, Rispail P, Tramini P. Evaluation of a new test for candidiasis diagnosis in elderly people. Odontostomatol Trop 2012; 35:37-43.

39. Kong EF, Kucharíková S, Van Dijck P, Peters BM, Shirtliff ME, Jabra-Rizk MA. Clinical implications of oral candidiasis: host tissue damage and disseminated bacterial disease. Infect Immun 83: 604-13.

40. Dineshshankar J, Sivakumar M, Karthikeyan M, Udayakumar P, Shanmugam KT, Kesavan G. Immunology of oral candidiasis. J Pharm Bioallied Sci 2014;6(Suppl 1):S9-S12.

41. García-Cuesta C, Sarrion-Pérez MG, Bagán JV. Current treatment of oral candidiasis: A literature review. J Clin Exp Dent 2014;6:576-82.

42. Beiro Fuentes R. Candidiasis oral experimental: estudio comparativo de la eficacia terapéutica de distintos excipientes asociados a la nistatina. Tesis Doctoral. Santiago de Compostela. 1998.

43. Park JB, Prodduturi S, Morott J, Kulkarni VI, Jacob MR, Khan SI, et al. Development of an antifungal denture adhesive film for oral candidiasis utilizing hot melt extrusion technology. Expert Opin Drug Deliv 2015;12:1-13.

44. Lalla RV, Patton LL, Dongari-Bagtzoglou A. Oral candidiasis: pathogenesis, clinical presentation, diagnosis and treatment strategies. J Calif Dent Assoc 2013;41:263-8.

45. Sheikh S, Gupta D, Pallagatti S, Singla I, Gupta $\mathrm{R}$, Goel V. Role of topical drugs in treatment of oral mucosal diseases. A literature review. N Y State Dent J 2013;79:58-64.

46. Nirmala MJ, Mukherjee A, Chandrasekaran $\mathrm{N}$. Improved efficacy of fluconazole against candidiasis using bio-based microemulsion technique. Biotechnol Appl Biochem 2013;60:417-29.

47. Sanitá PV, Mima EG, Pavarina AC, Jorge JH, Machado AL, Vergani CE. Susceptibility profile of a Brazilian yeast stock collection of Candida species isolated 
from subjects with Candida-associated denture stomatitis with or without diabetes. Oral Surg Oral Med Oral Pathol Oral Radiol 2013;116:562-9.

48. Gligorov J, Bastit L, Gervais H, Henni M, Kahila W, Lepille D, et al. Candidoscope Study Group. Prevalence and treatment management of oropharyngeal candidiasis in cancer patients: results of the French CANDIDOSCOPE study. Int J Radiat Oncol Biol Phys 2011;80:532-9.

49. Cotton MF, Ransome OJ. Oral ketoconazole and flucytosine for neonatal systemic candidiasis. S Afr Med J 1989;75:388-9.
50. Brito GN, Inocêncio AC, Querido SM, Jorge AO, Koga-Ito CY. In vitro antifungal susceptibility of Candida spp. oral isolates from HIV-positive patients and control individuals. Braz Oral Res 2011;25:28-33.

\section{CORRESPONDENCIA}

Dr. Andrés Blanco Carrión

República de El Salvador, $4,1^{\circ} \mathrm{C}$ 15702 Santiago de Compostela (A Coruña)

Correo electrónico: andres.blanco@usc.es 